\title{
Erratum to: Graphical tool of sparse factor analysis
}

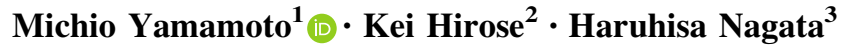

\section{Erratum to: Behaviormetrika \\ DOI 10.1007/s41237-016-0007-3}

Due to an unfortunate mistake at the typesetter's end, there was a typographical error on the 15th page of the article.

Line 2, The word SPMR should be SRMR.

The original article was corrected.

The online version of the original article can be found under doi:10.1007/s41237-016-0007-3.

\section{Michio Yamamoto}

michyama@kuhp.kyoto-u.ac.jp

1 Kyoto University, Kyoto, Japan

2 Kyushu University, Fukuoka, Japan

3 Osaka University, Osaka, Japan 